\title{
Steady compressible heat-conductive fluid with inflow boundary condition
}

\section{Chunhui Zhou*(1)}

\section{"Correspondence:}

zhouchunhui@seu.edu.cn Department of Mathematics, Southeast University, Nanjing, 210096, China

\section{Springer}

\begin{abstract}
In this paper, we study strong solutions to the steady compressible heat-conductive fluid near a non-zero constant flow with the Dirichlet boundary condition for the velocity on the inflow and outflow part of the boundary. We also consider the Dirichlet boundary condition for the temperature, and we do not need the thermal conductivity coefficient $\boldsymbol{\kappa}$ to be large. The existence of strong solutions is established for any Reynolds number and Mach number in the framework of perturbation.
\end{abstract}

MSC: $35 \mathrm{Q} 30 ; 76 \mathrm{~N} 10$

Keywords: steady fluid; compressible heat-conductive fluid; inflow boundary condition

\section{Introduction}

We will study strong solutions to the steady compressible heat-conductive fluid near a non-zero constant flow in a 2-D finite channel $\Omega=(0,1) \times(-1,1)$. As far as the internal friction (viscosity) and thermal conduction are concerned, the complete system of equations of steady compressible fluid can be expressed by

$$
\begin{aligned}
& \operatorname{div}(\rho \mathbf{u})=0, \\
& \operatorname{div}(\rho \mathbf{u} \otimes \mathbf{u})+\nabla p=\operatorname{div} \mathbb{S}+\rho \mathbf{f}+\mathbf{g}, \\
& \operatorname{div}((E+p) \mathbf{u})+\operatorname{div} \mathbf{q}=\operatorname{div}(\mathbb{S} \mathbf{u}) .
\end{aligned}
$$

Here, $\rho$ and $\mathbf{u}=\left(u_{1}, u_{2}\right)$ denote the density and velocity of the fluid, respectively, $E:=$ $\frac{1}{2} \rho|\mathbf{u}|^{2}+\rho e$ denotes the total energy, $e$ is the specific internal energy, and $\mathbf{q}$ is the heat flux, $p$ is the pressure, $\mathbf{f}=\left(f_{1}, f_{2}\right)$ and $\mathbf{g}=\left(g_{1}, g_{2}\right)$ denote the external volume and non-volume forces, $\mathbb{S}$ denotes the viscous stress tensor. In this paper, we will consider the viscous stress tensor in the form of a Newtonian fluid:

$$
\mathbb{S}=\mu\left(\nabla \mathbf{u}+\nabla \mathbf{u}^{T}\right)+\lambda \operatorname{div} \mathbf{u} \mathbb{I},
$$

where $\mu$ and $\lambda$ are constant viscosity coefficients which have to satisfy thermodynamic constraints $\mu>0, \lambda+2 \mu / N \geq 0$, and $\frac{1}{2}\left(\nabla \mathbf{u}+\nabla \mathbf{u}^{T}\right)$ is the deformation tensor. We will

(c) The Author(s) 2017. This article is distributed under the terms of the Creative Commons Attribution 4.0 International License (http://creativecommons.org/licenses/by/4.0/), which permits unrestricted use, distribution, and reproduction in any medium, provided you give appropriate credit to the original author(s) and the source, provide a link to the Creative Commons license, and indicate if changes were made. 
consider the physical situation where $e, p$ satisfy the equations of state

$$
e=c_{V} \theta, \quad p=R \rho \theta \quad \text { (polytropic ideal gas) }
$$

$R$ and $c_{V}$ are positive constants. The heat flux obeys the Fourier law: $\mathbf{q}=-\kappa \nabla \theta$ with $\kappa$ being a positive constant.

Let $\mathbf{n}$ be the outward unit normal of $\partial \Omega$, we are interested in the case when $\mathbf{u}$ is a small perturbation around $U_{0}=(1,0)$, thus $\partial \Omega$ is divided into the inflow part $\Gamma_{\text {in }}=\left\{x_{1}=0,0 \leq\right.$ $\left.x_{2} \leq 1\right\}(\mathbf{u} \cdot \mathbf{n}<0)$, the outflow part $\Gamma_{\text {out }}=\left\{x_{1}=1,0 \leq x_{2} \leq 1\right\}(\mathbf{u} \cdot \mathbf{n}>0)$, and the impermeable wall $\Gamma_{0}=\left\{0 \leq x_{1} \leq 1, x_{2}=0\right\} \cup\left\{0 \leq x_{1} \leq 1, x_{2}=1\right\}(\mathbf{u} \cdot \mathbf{n}=0)$. For simplicity, we will consider the case when $\mathbf{f}=\mathbf{g}=0$ in (1.2), and we point out that our result still holds for small $\mathbf{f}$ and $\mathbf{g}$. More explicitly, we will study the following system in this paper:

$$
\begin{aligned}
& \operatorname{div}(\rho \mathbf{u})=0, \quad \text { in } \Omega, \\
& \rho \mathbf{u} \cdot \nabla \mathbf{u}+\nabla p-\operatorname{div} \mathbb{S}(\nabla \mathbf{u})=0, \quad \text { in } \Omega, \\
& C_{V} \rho \mathbf{u} \cdot \nabla \theta+p \operatorname{div} \mathbf{u}=\kappa \Delta \theta+\Psi, \quad \text { in } \Omega, \\
& \mathbf{u}=\mathbf{u}_{0}, \quad \text { on } \Gamma_{\text {in }} \cup \Gamma_{\text {out }}, \\
& \rho=\rho_{\text {in }}, \quad \text { on } \Gamma_{\text {in }}, \\
& \operatorname{curl} \mathbf{u}=\mathbf{u} \cdot \mathbf{n}=0, \quad \text { on } \Gamma_{0}, \\
& \theta=\theta_{0}, \quad \text { on } \partial \Omega .
\end{aligned}
$$

$\Psi=2 \mu(\nabla \mathbf{u}: \nabla \mathbf{u})+\lambda(\operatorname{div} \mathbf{u})^{2}$ is the dissipation function.

There is rich literature dealing with the theory of steady compressible Navier-Stokes equations. Concerning the global existence of weak solutions to both compressible isentropic fluids and compressible heat-conductive fluids with large force, one can refer to [1-10]. We mention here that the studies above on the existence of weak solutions are all under the condition $\mathbf{u} \cdot \mathbf{n}=0$ on the boundary, i.e., the fluid cannot penetrate the wall. If $\mathbf{u} \cdot \mathbf{n} \neq 0$ on some part of the boundary, the existence of stationary weak solutions is essentially an open problem. The existence of strong solutions near equilibrium with different boundary or free boundary value problem also was investigated in [11-19], for example, where the linearized system of the Navier-Stokes equations can be viewed as a perturbation around the Stokes system. Considering the steady compressible fluids with inhomogeneous boundary conditions, the authors in [20-23] study the existence and regularity of compressible fluids with inflow boundary conditions in different domains when the Reynolds number is small. When the characteristic set on the boundary satisfies a certain assumption, the authors in $[24,25]$ can show the well-posedness to both isentropic fluids and heat-conductive fluids containing inflow boundary with small Reynolds number, but not infinitesimally small, and small Mach number.

In [26, 27], without the smallness assumption of the Reynolds number and the Mach number, the authors can obtain the strong solutions to both isentropic fluids and heatconductive fluids under inhomogeneous slip boundary conditions when friction coefficient is large on the boundary. Recently, the authors in [28] obtained the existence of strong solutions to steady compressible isentropic fluids near a uniform non-zero constant flow in a 2-D channel under the Dirichlet boundary condition for the velocity on the inflow and 
outflow part of the boundary without the smallness assumption of both Reynolds number and the Mach number.

In this paper, we will take into consideration thermal conduction and study strong solutions near a non-zero constant flow with the Dirichlet boundary condition for the velocity on the inflow and outflow part of the boundary. For simplicity, we will consider the Dirichlet boundary condition for the temperature. Here we do not need the Reynolds number and the Mach number to be small, and do not need the thermal conductivity coefficient $\kappa$ to be large.

We define

$$
E_{0}=\left|\mathbf{u}_{0}-U_{0}\right|_{2-1 / p, p ; \Gamma_{\text {in }}} \cup \Gamma_{\text {out }}+\left|\rho_{\text {in }}-1\right|_{1, p ; \Gamma_{\text {in }}}+\left|\theta_{0}-1\right|_{2-1 / p, p ; \partial \Omega} .
$$

Our main result reads as follows.

Theorem 1.1 Let $E_{0}$ defined in (1.6) be small enough, $\mathbf{u}_{0} \cdot \mathbf{n}=0$ on $\Gamma_{0}$ and $2<p<\infty$, then system $(1.5)$ is uniquely solvable in $W^{2, p}(\Omega) \times W^{1, p}(\Omega) \times W^{2, p}(\Omega)$ and the solution $(\mathbf{u}, \rho, \theta)$ satisfies

$$
\left\|\mathbf{u}-U_{0}\right\|_{2, p ; \Omega}+\|\rho-1\|_{1, p ; \Omega}+\|\theta-1\|_{2, p ; \Omega} \leq C(p, \Omega) E_{0} .
$$

Notation: We use standard notation for the function spaces, i.e., we denote by $L^{p}(\Omega)$, $W^{k, p}(\Omega)(p \geq 1)$ the Lebesgue spaces and the Sobolev spaces, respectively, by $H^{k}(\Omega)$ the Hilbert space, by $|\cdot|$ the Lebesgue norm at the boundary, and by $\|\cdot\|$ the Lebesgue norm in $\Omega$. Besides, we denote

$$
V=\left\{\mathbf{u} \in H^{1}(\Omega) \mid \mathbf{u}=0 \text { on } \Gamma_{\text {in }} \cup \Gamma_{\text {out }}, \mathbf{u} \cdot \mathbf{n}=0 \text { on } \Gamma_{0}\right\} .
$$

The proof of Theorem 1.1 is based on the a priori uniform estimates of the approximate solutions in Hilbert spaces as well as the improved $L_{p}(p>2)$ estimates of the solutions to the linearized equations. Comparing with the case of the compressible isentropic fluids in [28], here the pressure in momentum equation depends on both density and the temperature, while the temperature is governed by an elliptic equation. We shall consider the mixed system together and prove the existence of the approximate solutions in Hilbert spaces by the a priori uniform estimates and the Leray-Schauder fixed point theorem. To deal with the nonlinear system, we need the improved $L_{p}(p>2)$ estimates of the solutions to the linearized equations. We first prove the $H^{2}(\Omega)$ estimate of the temperature by the theory of elliptic equations. Then, by the result of the $L_{p}(p>2)$ estimates of the mass equation and the momentum equations in [28], one has $(\mathbf{u}, \rho, \theta) \in W^{2, p}(\Omega) \times W^{1, p}(\Omega) \times W^{2, p}(\Omega)$. Finally, as the term $\hat{\mathbf{u}} \cdot \nabla \rho$ brings the loss of regularity in the linearized transport equation, we can only obtain the existence of weak solutions to the original nonlinear system in the first step, and then the regularity of the weak solutions follows from the a priori uniform estimate above.

The rest of this paper is organized as follows. In Section 2, we derive the equivalent system for the perturbations, i.e., the linearized system for system (1.5). In Section 3, we first obtain the weak solutions to the linearized system by taking to the limit of an approximate system containing a viscous term in the transport equation. Then we prove that the weak 
solutions are also strong solutions to the linear system (1.5). Finally, in Section 4 we prove our main result Theorem 1.1.

\section{Perturbations and linearization of the problem}

As Theorem 1.1 concerns the problem of small perturbations around a constant flow, it will be convenient to write the equivalent system for the perturbations $(\overline{\mathbf{u}}, \bar{\rho}, \bar{\theta})$. First, by the theory of elliptic equations of second order, we can find $\tilde{\mathbf{u}}, \tilde{\theta} \in W^{2, p}(\Omega)$ satisfying

$$
\begin{aligned}
& \tilde{\mathbf{u}}=\mathbf{u}_{0}-U_{0} \quad \text { on } \Gamma_{\text {in }} \cup \Gamma_{\text {out }}, \quad \tilde{\mathbf{u}} \cdot \mathbf{n}=\operatorname{curl} \tilde{\mathbf{u}}=0 \quad \text { on } \Gamma_{0}, \\
& \|\tilde{\mathbf{u}}\|_{2, p ; \Omega} \leq C(p)\left|\mathbf{u}_{0}-U_{0}\right|_{2-1 / p, p ; \Gamma_{\text {in }} \cup \Gamma_{\text {out }}}, \\
& \tilde{\theta}=\theta_{0}-1 \quad \text { on } \partial \Omega, \quad\|\tilde{\theta}\|_{2, p ; \Omega} \leq C(p)\left|\theta_{0}-1\right|_{2-1 / p, p ; \Gamma_{\text {in }} \cup \Gamma_{\text {out }} .} .
\end{aligned}
$$

Then denote

$$
\overline{\mathbf{u}}=\mathbf{u}-\tilde{\mathbf{u}}-U_{0}, \quad \bar{\rho}=\rho-1, \quad \bar{\theta}=\theta-\tilde{\theta}-1 .
$$

We arrive at the equivalent system for the perturbations $(\overline{\mathbf{u}}, \bar{\rho}, \bar{\theta})$ :

$$
\begin{aligned}
& \partial_{1} \bar{\rho}+(\overline{\mathbf{u}}+\tilde{\mathbf{u}}) \cdot \nabla \bar{\rho}+\operatorname{div} \overline{\mathbf{u}}=F(\overline{\mathbf{u}}, \bar{\rho}) \quad \text { in } \Omega, \\
& \partial_{1} \overline{\mathbf{u}}+\nabla \bar{\rho}+\nabla \bar{\theta}-\operatorname{div} \mathbb{S}(\nabla \overline{\mathbf{u}})=G(\overline{\mathbf{u}}, \bar{\rho}, \bar{\theta}) \quad \text { in } \Omega, \\
& \partial_{1} \bar{\theta}+\operatorname{div} \overline{\mathbf{u}}-\kappa \Delta \bar{\theta}=H(\overline{\mathbf{u}}, \bar{\rho}, \bar{\theta}) \quad \text { in } \Omega, \\
& \bar{\rho}=\rho_{\text {in }}-1 \quad \text { on } \Gamma_{\text {in }}, \\
& \overline{\mathbf{u}}=0 \quad \text { on } \Gamma_{\text {in }} \cup \Gamma_{\text {out }}, \\
& \overline{\mathbf{u}} \cdot \mathbf{n}=\operatorname{curl} \overline{\mathbf{u}}=0 \quad \text { on } \Gamma_{0}, \\
& \bar{\theta}=0 \quad \text { on } \partial \Omega,
\end{aligned}
$$

where

$$
\begin{aligned}
F(\overline{\mathbf{u}}, \bar{\rho})=- & \operatorname{div} \tilde{\mathbf{u}}-\bar{\rho} \operatorname{div}(\overline{\mathbf{u}}+\tilde{\mathbf{u}}), \\
G(\overline{\mathbf{u}}, \bar{\rho}, \bar{\theta})= & \operatorname{div} \mathbb{S}(\nabla \overline{\mathbf{u}})-(\bar{\rho}+1)\left(\overline{\mathbf{u}}+\tilde{\mathbf{u}}+U_{0}\right) \cdot \nabla \tilde{\mathbf{u}}-(\bar{\rho}+1)(\overline{\mathbf{u}}+\tilde{\mathbf{u}}) \cdot \nabla \overline{\mathbf{u}} \\
& -\bar{\rho} U_{0} \cdot \nabla \overline{\mathbf{u}}+R \nabla \tilde{\theta}+R \nabla \bar{\rho}(\bar{\theta}+\tilde{\theta}), \\
H(\overline{\mathbf{u}}, \bar{\rho}, \bar{\theta})= & \kappa \Delta \tilde{\theta}-(\bar{\rho}+1)\left(\overline{\mathbf{u}}+\tilde{\mathbf{u}}+U_{0}\right) \cdot \nabla \tilde{\theta}-(\bar{\rho}+1)(\overline{\mathbf{u}}+\tilde{\mathbf{u}}) \cdot \nabla \bar{\theta}-\bar{\rho} U_{0} \cdot \nabla \bar{\theta} \\
& -(\bar{\rho}+1)(\bar{\theta}+\tilde{\theta}) \operatorname{div}(\overline{\mathbf{u}}+\tilde{\mathbf{u}})-(\bar{\rho}+1) \operatorname{div} \tilde{\mathbf{u}} \\
& +2 \mu D(\overline{\mathbf{u}}+\tilde{\mathbf{u}}): D(\overline{\mathbf{u}}+\tilde{\mathbf{u}})+\lambda(\operatorname{div}(\overline{\mathbf{u}}+\tilde{\mathbf{u}}))^{2} .
\end{aligned}
$$

Before treating the nonlinear system (2.2), we will first establish the existence result to the following linear system:

$$
\begin{aligned}
& \partial_{1} \rho+\hat{\mathbf{u}} \cdot \nabla \rho+\operatorname{div} \mathbf{u}=f \quad \text { in } \Omega, \\
& \partial_{1} \mathbf{u}+\nabla \rho+\nabla \theta-\operatorname{div} \mathbb{S}(\nabla \mathbf{u})=g \quad \text { in } \Omega, \\
& \partial_{1} \theta+\operatorname{div} \mathbf{u}-\kappa \Delta \theta=h \quad \text { in } \Omega,
\end{aligned}
$$




$$
\begin{aligned}
& \rho=\rho_{0} \quad \text { on } \Gamma_{\text {in }}, \\
& \mathbf{u}=0 \quad \text { on } \Gamma_{\text {in }} \cup \Gamma_{\text {out }}, \\
& \mathbf{u} \cdot \mathbf{n}=\operatorname{curl} \mathbf{u}=0 \quad \text { on } \Gamma_{0}, \\
& \theta=0 \quad \text { on } \partial \Omega .
\end{aligned}
$$

\section{Solutions to the linearized system}

As the term $\hat{\mathbf{u}} \cdot \nabla \rho$ in the transport equation (2.4) brings the loss of regularity, it seems impossible to establish the existence result to system (2.4)-(2.10) by the fixed point theory. Instead, in the first step, we obtain the existence of weak solutions by taking the limit of its viscous solutions. Then, by the theory of elliptic equations and elliptic systems, we can prove that the weak solution $(\mathbf{u}, \rho, \theta)$ is in $W^{2, p}(\Omega) \times W^{1, p}(\Omega) \times W^{2, p}(\Omega)$.

\subsection{Preliminaries}

Definition 3.1 (Weak solution) We say that the triple $(\mathbf{u}, \rho, \theta) \in H^{1}(\Omega) \times L^{2}(\Omega) \times H^{1}(\Omega)$ is a weak solution to system (2.4)-(2.10) if it satisfies:

(1) for $\forall \varphi \in C^{1}(\bar{\Omega})$ with $\left.\varphi\right|_{\Gamma_{\text {out }}}=0$,

$$
-\int_{\Gamma_{\mathrm{in}}} \rho_{0}(s) \varphi(0, s) d s+\int_{\Omega}\left[\varphi \operatorname{div} u-\rho \partial_{1} \varphi-\rho \operatorname{div}(\varphi \hat{u})\right] d x=\int_{\Omega} f \varphi d x
$$

(2) for $\forall \phi \in V$,

$$
\int_{\Omega}\left[\partial_{1} \mathbf{u} \phi+\nabla \theta \phi-\rho \operatorname{div} \phi\right] d x+\int_{\Omega}[2 \mu D(\mathbf{u}): D(\phi)+\lambda \operatorname{div} u \operatorname{div} \phi] d x=\int_{\Omega} g \phi d x ;
$$

(3) for $\forall \psi \in H_{0}^{1}(\Omega)$,

$$
\int_{\Omega}\left[\partial_{1} \theta \psi+\operatorname{div} \mathbf{u} \psi+\kappa \nabla \theta \cdot \nabla \phi\right] d x=\int_{\Omega} h \psi d x
$$

Next, we turn to considering the following boundary problem:

$$
\begin{aligned}
& \partial_{1} \mathbf{u}-\mu \Delta \mathbf{u}-\lambda \nabla \operatorname{div} \mathbf{u}=G \quad \text { in } \Omega, \\
& \mathbf{u}=0 \quad \text { on } \Gamma_{\text {in }} \cup \Gamma_{\text {out }}, \\
& \mathbf{u} \cdot \mathbf{n}=\operatorname{curl} \mathbf{u}=0 \quad \text { on } \Gamma_{0} .
\end{aligned}
$$

Lemma 3.2 Let $\mathbf{u}=\left(u_{1}, u_{2}\right) \in H^{1}(\Omega)$ be the weak solution of system (3.4)-(3.6) in the domain $\Omega=(0,1) \times(-1,1)$. Then we have $\mathbf{u} \in H^{2}(\Omega)$, and it holds that

$$
\|\mathbf{u}\|_{2,2 ; \Omega} \leq\|G\|_{2 ; \Omega} .
$$

Proof To prove Lemma 3.2, we only need to prove the $H^{2}$ regularity of $\mathbf{u}$ near the corners of the domain.

Let $\mathbf{u}=\left(u_{1}, u_{2}\right)$ be the weak solution of system (3.4)-(3.6). Then, for any subdomain $D \subset \Omega$ away from the corners, it holds that $\mathbf{u} \in H^{2}(D)$, i.e., (3.4) holds a.e. in $\Omega$. Let $\phi(t) \in$ $C^{\infty}(\mathbb{R})$ be a cutoff function satisfying $\phi(t)=1$ when $|t| \leq 3 / 2, \phi(t)=0$ when $|t| \geq 2$ and 
$|\phi(t)| \leq 1$ in $\mathbb{R}$. Assume that $G_{1}^{*}, u_{1}^{*}$ are the even extensions of $G_{1}, u_{1}$ with respect to $x_{2}=$ 0,$1 ; u_{2}^{*}, G_{2}^{*}$ are the odd extensions of $u_{2}, G_{2}$ with respect to $x_{2}=0,1$, then $\mathbf{u}^{\prime} \doteq \phi\left(x_{2}\right) \mathbf{u}^{*}=$ $\left(\phi\left(x_{2}\right) u_{1}^{*}, \phi\left(x_{2}\right) u_{2}^{*}\right)$ satisfy the following elliptic system a.e. in $\Omega^{\prime}=(0,1) \times(-2,2)$ :

$$
\begin{aligned}
& -\mu \Delta \mathbf{u}^{\prime}-\lambda \nabla \operatorname{div} \mathbf{u}^{\prime}=G^{\prime} \text { in } \Omega^{\prime}, \\
& \mathbf{u}^{\prime}=0 \quad \text { on } \partial \Omega^{\prime}
\end{aligned}
$$

with $G^{\prime}=G-\partial_{1} \mathbf{u}$ in $\Omega$, and

$$
\begin{aligned}
& G_{1}^{\prime}=\phi\left(x_{2}\right) G_{1}^{*}-\lambda \phi^{\prime} u_{2}^{*}-\mu\left(\phi^{\prime \prime} u_{1} *+2 \phi^{\prime} u_{1} *\right), \\
& G_{2}^{\prime}=\phi\left(x_{2}\right) G_{2}^{*}-\lambda \phi^{\prime} u_{1}^{*}-(\mu+\lambda)\left(\phi^{\prime \prime} u_{2} *+2 \phi^{\prime} u_{2} *\right)
\end{aligned}
$$

in $\Omega^{\prime} \backslash \Omega$. Finally, (3.7) follows from the interior estimate of an elliptic system immediately.

\subsection{Weak solutions to the linear system}

The main result of this section is the following theorem.

Theorem 3.3 Let $\hat{\mathbf{u}} \in W^{2, p}(\Omega)$ satisfying $\hat{\mathbf{u}} \cdot \mathbf{n}=0$ on $\partial \Omega$ and $\|\hat{\mathbf{u}}\|_{2, p ; \Omega}$ be small enough. Then, for any $\rho_{0} \in H^{1}((0,1)), f \in H^{1}(\Omega), h, g \in L^{2}(\Omega)$, there exists a weak solution $(\mathbf{u}, \rho, \theta)$ to system (2.4)-(2.10) such that

$$
\|\rho\|_{L^{2}(\Omega)}+\|u\|_{1,2 ; \Omega}+\|\theta\|_{1,2 ; \Omega} \leq C\left(\left|\rho_{0}\right|_{1,2 ;(0,1)}+\|f\|_{L^{2}(\Omega)}+\|g\|_{H^{-1}(\Omega)}+\|h\|_{H^{-1}(\Omega)}\right),
$$

where $C=C(\mu, \lambda, \gamma, \kappa)$.

Proof To prove Theorem 3.3, we first get rid of the term $\hat{\mathbf{u}} \cdot \nabla \rho$ using a change of variables satisfying the identity

$$
\partial_{\bar{x}_{1}}=\partial_{x_{1}}+\hat{\mathbf{u}} \cdot \nabla_{x} .
$$

To this end, we define $\Pi: \Omega \rightarrow \hat{\Omega}$ by

$$
\left\{\begin{array}{l}
x_{1}=\bar{x}_{1}, \\
x_{2}=\bar{x}_{2}+\int_{0}^{\bar{x}_{1}} \frac{\hat{u}_{2}}{1+\hat{u}_{1}}\left(s, x_{2}\left(s, \bar{x}_{2}\right)\right) d s .
\end{array}\right.
$$

Then direct computation shows that $\hat{\Omega}=(0,1) \times(-1,1)$ and

$$
\left\|\frac{\partial\left(\bar{x}_{1}, \bar{x}_{2}\right)}{\partial\left(x_{1}, x_{2}\right)}-I\right\|_{1, p ; \Omega} \leq\|\hat{\mathbf{u}}\|_{2, p ; \Omega}
$$

where $I$ is the unit matrix. The equivalent system in the new coordinates is as follows (we have omitted the superscript in the absence of confusion):

$$
\partial_{1} \rho+\operatorname{div} \mathbf{u}=f+\tilde{f}(\mathbf{u}) \quad \text { in } \Omega
$$




$$
\begin{aligned}
& \partial_{1} \mathbf{u}+\nabla \rho+\nabla \theta-\mu \Delta \mathbf{u}-\lambda \nabla \operatorname{div} \mathbf{u}=g+\tilde{g}(\mathbf{u})+\hat{g}(\rho, \theta) \quad \text { in } \Omega, \\
& \partial_{1} \theta+\operatorname{div} \mathbf{u}-\kappa \Delta \theta=h+\tilde{h}(\theta)+\hat{h}(\mathbf{u}) \quad \text { in } \Omega, \\
& \rho=\rho_{0} \quad \text { on } \Gamma_{\text {in }} \\
& \mathbf{u}=0 \quad \text { on } \Gamma_{\text {in }} \cup \Gamma_{\text {out }}, \\
& \mathbf{u} \cdot \mathbf{n}=\operatorname{curl} \mathbf{u}=0 \quad \text { on } \Gamma_{0} \\
& \theta=0 \quad \text { on } \partial \Omega,
\end{aligned}
$$

where $\hat{\Gamma}_{\text {in }}=\Pi^{-1}\left(\Gamma_{\text {in }}\right), \hat{\Gamma}_{\text {out }}=\Pi^{-1}\left(\Gamma_{\text {out }}\right), \hat{\Gamma}_{0}=\Pi^{-1}\left(\Gamma_{0}\right), \hat{g}=\left(\hat{g}_{1}, \hat{g}_{2}\right), \tilde{g}=\left(\tilde{g}_{1}, \tilde{g}_{2}\right)$ and

$$
\begin{aligned}
& \tilde{f}(\mathbf{u})=-\partial_{\bar{x}_{2}} u_{1} \frac{\partial \bar{x}_{2}}{\partial x_{1}}-\left(\frac{\partial \bar{x}_{2}}{\partial x_{2}}-1\right) \partial_{\bar{x}_{2}} u_{2}+\frac{\hat{u}_{1}}{1+\hat{u}_{1}}\left(\partial_{\bar{x}_{1}} u_{1}+\partial_{\bar{x}_{2}} u_{1} \frac{\partial \bar{x}_{2}}{\partial x_{1}}+\frac{\partial \bar{x}_{2}}{\partial x_{2}} \partial_{\bar{x}_{2}} u_{2}-f\right), \\
& \hat{g}_{1}(\rho, \theta)=-\partial_{\bar{x}_{2}}(\rho+\theta) \frac{\partial \bar{x}_{2}}{\partial x_{1}} \\
& \hat{g}_{2}(\rho, \theta)=-\partial_{\bar{x}_{2}}(\rho+\theta)\left(\frac{\partial \bar{x}_{2}}{\partial x_{2}}-1\right), \\
& \tilde{g}_{1}(\mathbf{u})=-\partial_{\bar{x}_{2}} u_{1} \frac{\partial \bar{x}_{2}}{\partial x_{1}}-2(\mu+\lambda) \partial_{\bar{x}_{1} \bar{x}_{2}} u_{1} \frac{\partial \bar{x}_{2}}{\partial x_{1}}-\partial_{\bar{x}_{2}} u_{1}\left[\mu \Delta_{x} \bar{x}_{2}+\lambda \frac{\partial^{2} \bar{x}_{2}}{\partial x_{1}^{2}}\right] \\
& -\lambda\left[\partial_{\bar{x}_{1} \bar{x}_{2}} u_{2}\left(\frac{\partial \bar{x}_{2}}{\partial x_{2}}-1\right)+\partial_{\bar{x}_{2}}^{2} u_{2} \frac{\partial \bar{x}_{2}}{\partial x_{1}} \frac{\partial \bar{x}_{2}}{\partial x_{2}}+\partial_{\bar{x}_{2}} u_{2} \frac{\partial^{2} \bar{x}_{2}}{\partial x_{1} \partial x_{2}}\right] \\
& -\left((\mu+\lambda)\left(\frac{\partial \bar{x}_{2}}{\partial x_{1}}\right)^{2}+\mu\left[\left(\frac{\partial \bar{x}_{2}}{\partial x_{2}}\right)^{2}-1\right]\right) \partial_{\bar{x}_{2}}^{2} u_{1} \\
& \tilde{g}_{2}(\mathbf{u})=g_{2}-\partial_{\bar{x}_{2}} u_{2} \frac{\partial \bar{x}_{2}}{\partial x_{1}}-2 \mu \partial_{\bar{x}_{1} \bar{x}_{2}} u_{2} \frac{\partial \bar{x}_{2}}{\partial x_{1}}-\partial_{\bar{x}_{2}} u_{2}\left[\mu \Delta_{x} \bar{x}_{2}+\lambda \frac{\partial^{2} \bar{x}_{2}}{\partial x_{2}^{2}}\right] \\
& -\lambda\left[\partial_{\bar{x}_{1} \bar{x}_{2}} u_{1}\left(\frac{\partial \bar{x}_{2}}{\partial x_{2}}-1\right)+\partial_{\bar{x}_{2}}^{2} u_{1} \frac{\partial \bar{x}_{2}}{\partial x_{1}} \frac{\partial \bar{x}_{2}}{\partial x_{2}}+\partial_{\bar{x}_{2}} u_{1} \frac{\partial^{2} \bar{x}_{2}}{\partial x_{1} \partial_{x_{2}}}\right] \\
& -\partial_{\bar{x}_{2}}^{2} u_{2}\left(\mu\left(\frac{\partial \bar{x}_{2}}{\partial x_{1}}\right)^{2}+(\mu+\lambda)\left[\left(\frac{\partial \bar{x}_{2}}{\partial x_{2}}\right)^{2}-1\right]\right) \text {, } \\
& \hat{h}(\mathbf{u})=-\partial_{\bar{x}_{2}} u_{1} \frac{\partial \bar{x}_{2}}{\partial x_{1}}-\left(\frac{\partial \bar{x}_{2}}{\partial x_{2}}-1\right) \partial_{\bar{x}_{2}} u_{2} \text {, } \\
& \tilde{h}(\theta)=-\partial_{\bar{x}_{2}} \theta \frac{\partial \bar{x}_{2}}{\partial x_{1}}-2 \mu \partial_{\bar{x}_{1} \bar{x}_{2}} \theta \frac{\partial \bar{x}_{2}}{\partial x_{1}}-\mu \partial_{\bar{x}_{2}} \theta \Delta_{x} \bar{x}_{2}-\mu\left[\left(\frac{\partial \bar{x}_{2}}{\partial x_{1}}\right)^{2}+\left(\frac{\partial \bar{x}_{2}}{\partial x_{2}}\right)^{2}-1\right] \partial_{\bar{x}_{2}}^{2} \theta \text {. }
\end{aligned}
$$

Next we consider the following approximate system to (3.11)-(3.17):

$$
\begin{aligned}
& \partial_{1} \rho_{\epsilon}-\epsilon \Delta \rho_{\epsilon}+\operatorname{div} \mathbf{u}_{\epsilon}=f+\tilde{f}\left(\mathbf{u}_{\epsilon}\right) \quad \text { in } \hat{\Omega}, \\
& \partial_{1} \mathbf{u}_{\epsilon}+\nabla \rho_{\epsilon}+\nabla \theta_{\epsilon}-\mu \Delta \mathbf{u}_{\epsilon}-\lambda \nabla \operatorname{div} \mathbf{u}_{\epsilon}=g+\tilde{g}\left(\mathbf{u}_{\epsilon}\right)+\hat{g}\left(\rho_{\epsilon}, \theta_{\epsilon}\right) \quad \text { in } \hat{\Omega}, \\
& \partial_{1} \theta_{\epsilon}+\operatorname{div} \mathbf{u}_{\epsilon}-\kappa \Delta \theta_{\epsilon}=h+\tilde{h}\left(\theta_{\epsilon}\right)+\hat{h}\left(\mathbf{u}_{\epsilon}\right) \quad \text { in } \Omega, \\
& \rho_{\epsilon}=\rho_{0} \quad \text { on } \hat{\Gamma}_{\text {in }}, \\
& \frac{\partial \rho_{\epsilon}}{\partial \mathbf{n}}=0 \quad \text { on } \hat{\Gamma}_{\text {out }} \cup \hat{\Gamma}_{0}, \\
& \mathbf{u}_{\epsilon}=0 \text { on } \hat{\Gamma}_{\text {in }} \cup \hat{\Gamma}_{\text {out }},
\end{aligned}
$$




$$
\begin{aligned}
& \mathbf{u}_{\epsilon} \cdot \mathbf{n}=\operatorname{curl} \mathbf{u}_{\epsilon}=0 \quad \text { on } \hat{\Gamma}_{0} . \\
& \theta_{\epsilon}=0 \quad \text { on } \partial \Omega .
\end{aligned}
$$

Here, $\epsilon>0$ is a small constant.

We define two operators $S_{1}: v \in H^{1}(\hat{\Omega}) \rightarrow \rho_{\epsilon} \in H^{1}(\hat{\Omega})$ and $S_{2}: v \in H^{1}(\hat{\Omega}) \rightarrow \theta_{\epsilon} \in H^{1}(\hat{\Omega})$ by letting $\rho_{\epsilon}=S_{1}(v)$ and $\theta_{\epsilon}=S_{2}(v)$ be the solutions of the following two elliptic problems:

$$
\begin{aligned}
& \partial_{1} \rho_{\epsilon}-\epsilon \Delta \rho_{\epsilon}=-\operatorname{div} v+f+\tilde{f}(v) \quad \text { in } \hat{\Omega}, \\
& \rho_{\epsilon}=\rho_{0} \quad \text { on } \hat{\Gamma}_{\text {in }} \\
& \frac{\partial \rho_{\epsilon}}{\partial \mathbf{n}}=0 \quad \text { on } \hat{\Gamma}_{\text {out }} \cup \hat{\Gamma}_{0}
\end{aligned}
$$

and

$$
\begin{aligned}
& \partial_{1} \theta_{\epsilon}-\kappa \Delta \theta_{\epsilon}+\tilde{h}\left(\theta_{\epsilon}\right)=-\operatorname{div} \nu+h+\hat{h}(v) \text { in } \hat{\Omega}, \\
& \theta=0 \quad \text { on } \partial \hat{\Omega} .
\end{aligned}
$$

As for $v \in H^{1}(\hat{\Omega})$ one has $-\operatorname{div} v+f+\tilde{f}(v),-\operatorname{div} v+h+\hat{h}(v) \in L^{2}(\hat{\Omega})$, then for any subdomain $D \subset \hat{\Omega}$ away from the corners, it holds that $\rho_{\epsilon}, \theta_{\epsilon} \in H^{2}(D)$, i.e., (3.19) and (3.22) hold a.e. in $\hat{\Omega}$.

To proceed further, we define the operator $T: v \mapsto \mathbf{u}_{\epsilon}$ by the following elliptic system:

$$
\begin{aligned}
& \partial_{1} \mathbf{u}_{\epsilon}-\mu \Delta \mathbf{u}_{\epsilon}-\lambda \nabla \operatorname{div} \mathbf{u}_{\epsilon}-\tilde{g}\left(\mathbf{u}_{\epsilon}\right) \\
& =-\nabla\left[S_{1}(v)+S_{2}(v)\right]+g+\hat{g}\left(S_{1}(v), S_{2}(v)\right) \text { in } \hat{\Omega}, \\
& \mathbf{u}_{\epsilon}=0 \text { on } \hat{\Gamma}_{\text {in }} \cup \hat{\Gamma}_{\text {out }} \text {, } \\
& \mathbf{u}_{\epsilon} \cdot \mathbf{n}=\operatorname{curl} \mathbf{u}_{\epsilon}=0 \quad \text { on } \hat{\Gamma}_{0} .
\end{aligned}
$$

We want to prove Theorem 3.3 by showing that $T$ has a fixed point in $H^{1}(\Omega)$.

First of all, since for any $v \in H^{1}(\hat{\Omega})$, the term on the right-hand side of (3.23) belongs to $L^{2}(\Omega)$, then $\mathbf{u}_{\epsilon} \in H^{2}(D)$ in any subdomain $D \subset \hat{\Omega}$ away from the corners. Then, by changing of variables $\Pi^{-1}: \hat{\Omega} \rightarrow \Omega$ and Lemma 3.2, one has $\mathbf{u}_{\epsilon} \in H^{2}(\hat{\Omega})$, which implies that $T$ is a compact mapping from $H^{1}$ into $H^{1}$.

Now, by Leray-Schauder fixed point theory ([29], Chapter 11), we only need to prove that there exists a constant $M$ such that for all $\mathbf{u} \in H^{1}$ and $\sigma \in[0,1]$ satisfying $\mathbf{u}=\sigma T \mathbf{u}$, it holds that

$$
\|\mathbf{u}\|_{H^{1}}<M
$$

i.e., for any $\sigma \in[0,1]$, the solutions to the problem

$$
\begin{aligned}
& \partial_{1} \mathbf{u}_{\epsilon}-\mu \Delta \mathbf{u}_{\epsilon}-\lambda \nabla \operatorname{div} \mathbf{u}_{\epsilon}-\tilde{g}\left(\mathbf{u}_{\epsilon}\right) \\
& \quad=\sigma\left[-\nabla\left[S_{1}\left(\mathbf{u}_{\epsilon}\right)+S_{2}\left(\mathbf{u}_{\epsilon}\right)\right]+g+\hat{g}\left(S_{1}\left(\mathbf{u}_{\epsilon}\right), S_{2}\left(\mathbf{u}_{\epsilon}\right)\right)\right] \text { in } \hat{\Omega}, \\
& \mathbf{u}_{\epsilon}=0 \quad \text { on } \hat{\Gamma}_{\text {in }} \cup \hat{\Gamma}_{\text {out }},
\end{aligned}
$$


$\mathbf{u}_{\epsilon} \cdot \mathbf{n}=\operatorname{curl} \mathbf{u}_{\epsilon}=0 \quad$ on $\hat{\Gamma}_{0}$

satisfy the uniform estimate (3.26).

To this end, we first test (3.19), (3.22) and (3.27) by $\rho_{\epsilon}, \theta_{\epsilon}, \mathbf{u}_{\epsilon}$, respectively, to obtain

$$
\begin{aligned}
& \frac{1}{2} \int_{0}^{1}\left[\rho_{\epsilon}^{2}\left(1, x_{2}\right)-\rho_{\epsilon}^{2}\left(0, x_{2}\right)\right] d x_{2}+\epsilon \int_{0}^{1} \rho_{\epsilon} \partial_{1} \rho_{\epsilon}\left(0, x_{2}\right) d x_{2} \\
& \quad+\epsilon \int_{\hat{\Omega}}\left|\nabla \rho_{\epsilon}\right|^{2} d x+\int_{\hat{\Omega}} \rho_{\epsilon} \operatorname{div} \mathbf{u}_{\epsilon} d x \\
& \quad=\int_{\hat{\Omega}} \rho_{\epsilon}\left(f+\tilde{f}\left(\mathbf{u}_{\epsilon}\right)\right) d x
\end{aligned}
$$

and

$$
\begin{gathered}
\int_{\hat{\Omega}} \theta_{\epsilon} \partial_{1} \theta_{\epsilon} d x+\int_{\hat{\Omega}} \theta_{\epsilon} \operatorname{div} \mathbf{u}_{\epsilon} d x+\kappa \int_{\hat{\Omega}}\left|\nabla \theta_{\epsilon}\right|^{2} d x \\
=\int_{\hat{\Omega}}\left(h+\hat{h}\left(\mathbf{u}_{\epsilon}\right)-\tilde{h}\left(\theta_{\epsilon}\right) \theta_{\epsilon} d x\right.
\end{gathered}
$$

and

$$
\begin{aligned}
-\sigma & \int_{\hat{\Omega}}\left(\rho_{\epsilon}+\theta_{\epsilon}\right) \operatorname{div} \mathbf{u}_{\epsilon} d x+\int_{\hat{\Omega}}\left[\mu\left|\nabla \mathbf{u}_{\epsilon}\right|^{2}+\lambda\left(\operatorname{div} \mathbf{u}_{\epsilon}\right)^{2}\right] d x \\
& =\int_{\hat{\Omega}}\left[\tilde{g}\left(\mathbf{u}_{\epsilon}\right)+\sigma\left(g+\hat{g}\left(\rho_{\epsilon}, \theta_{\epsilon}\right)\right)\right] \mathbf{u}_{\epsilon} d x .
\end{aligned}
$$

Besides, since (3.19) holds a.e. in $\hat{\Omega}$, it is convenient to multiply (3.19) by $\partial_{1} \rho_{\epsilon}$ to obtain

$$
\begin{aligned}
& \int_{\hat{\Omega}}\left(\partial_{1} \rho_{\epsilon}\right)^{2} d x+\frac{1}{2} \epsilon \int_{0}^{1}\left[\left(\partial_{1} \rho_{\epsilon}\right)^{2}\left(0, x_{2}\right)+\left(\partial_{2} \rho_{\epsilon}\right)^{2}\left(1, x_{2}\right)\right] d x_{2} \\
& =\frac{1}{2} \epsilon \int_{0}^{1}\left[\left(\partial_{2} \rho_{\epsilon}\right)^{2}\left(0, x_{2}\right) d x_{2}+\int_{\hat{\Omega}}\left[-\operatorname{div} \mathbf{u}_{\epsilon}+f+\tilde{f}\left(\mathbf{u}_{\epsilon}\right)\right] \partial_{1} \rho_{\epsilon} d x\right.
\end{aligned}
$$

which implies that

$$
\begin{aligned}
& \left\|\partial_{1} \rho_{\epsilon}\right\|_{L^{2}(\hat{\Omega})}+\sqrt{\epsilon}\left[\left|\partial_{1} \rho_{\epsilon}(0, \cdot)\right|_{L^{2}}+\left|\partial_{2} \rho_{\epsilon}(1, \cdot)\right|_{L^{2}}\right] \\
& \leq C\left(\|f\|_{L^{2}(\hat{\Omega})}+\left|\rho_{0}\right|_{H^{1}}+\left\|\mathbf{u}_{\epsilon}\right\|_{H^{1}}\right) .
\end{aligned}
$$

Combining (3.30)-(3.33), we can arrive at the following estimate:

$$
\begin{aligned}
& \sigma \epsilon \int_{\hat{\Omega}}\left|\nabla \rho_{\epsilon}\right|^{2} d x+\sigma \kappa \int_{\hat{\Omega}}\left|\nabla \theta_{\epsilon}\right|^{2} d x+\int_{\hat{\Omega}}\left|\nabla \mathbf{u}_{\epsilon}\right|^{2} d x+\sigma \epsilon \int_{0}^{1} \rho_{\epsilon} \partial_{1} \rho_{\epsilon}\left(0, x_{2}\right) d x_{2} \\
& \leq C\left(\delta\left\|\rho_{\epsilon}\right\|_{L^{2}}^{2}+\|g\|_{H^{-1}}^{2}+\left|\rho_{0}\right|_{2}^{2}\right) \\
& \quad+\sigma \int_{\hat{\Omega}}\left[\rho_{\epsilon}\left(f+\tilde{f}\left(\mathbf{u}_{\epsilon}\right)\right)+\left(h+\hat{h}\left(\mathbf{u}_{\epsilon}\right)-\tilde{h}\left(\theta_{\epsilon}\right) \theta_{\epsilon}\right] d x,\right.
\end{aligned}
$$

where $\delta>0$ is a small constant and $C=C(\mu, \lambda, \gamma, \kappa)$. 
Then it follows from (3.33) and (3.34) that

$$
\begin{aligned}
& \left\|\rho_{\epsilon}\right\|_{L^{2}(\hat{\Omega})}+\sigma \sqrt{\epsilon}\left\|\nabla \rho_{\epsilon}\right\|_{L^{2}}+\left\|\mathbf{u}_{\epsilon}\right\|_{1,2 ; \hat{\Omega}}+\left\|\theta_{\epsilon}\right\|_{1,2 ; \hat{\Omega}} \\
& \quad \leq C\left(\left|\rho_{0}\right|_{1,2 ; \hat{\Gamma}_{i n}}+\|f\|_{L^{2}(\hat{\Omega})}+\|g\|_{H^{-1}(\hat{\Omega})}+\|h\|_{H^{-1}(\hat{\Omega})}\right) .
\end{aligned}
$$

Thus by Leray-Schauder fixed point theory ([29], Chapter 11), there is a solution $\left(\rho_{\epsilon}, \mathbf{u}_{\epsilon}, \theta_{\epsilon}\right) \in L_{2}(\hat{\Omega}) \times H^{1}(\hat{\Omega}) \times H^{1}(\hat{\Omega})$ to system (3.18) satisfying (3.35). Moreover, (3.35) implies that there are $\rho \in L^{2}(\hat{\Omega}), \mathbf{u} \in V$ and $\theta \in H^{1}(\hat{\Omega})$ such that

$$
\begin{array}{ll}
\rho_{\epsilon} \rightarrow \rho & \text { weakly in } L^{2}(\hat{\Omega}) ; \quad \mathbf{u}_{\epsilon} \rightarrow \mathbf{u} \quad \text { weakly in } H^{1}(\hat{\Omega}) ; \\
\theta_{\epsilon} \rightarrow \theta \quad \text { weakly in } H^{1}(\hat{\Omega}), &
\end{array}
$$

and for any $\varphi \in H^{1}(\hat{\Omega})$,

$$
\left(\epsilon \Delta \rho_{\epsilon}, \varphi\right)=-\int_{\hat{\Omega}} \epsilon \nabla \rho_{\epsilon} \nabla \varphi d x \rightarrow 0, \quad \text { as } \epsilon \rightarrow 0,
$$

and

$$
\|\rho\|_{L^{2}(\hat{\Omega})}+\|\mathbf{u}\|_{1,2 ; \hat{\Omega}}+\|\theta\|_{1,2 ; \hat{\Omega}} \leq C\left(\left|\rho_{0}\right|_{1,2 ; \hat{\Gamma}_{\mathrm{in}}}+\|f\|_{L^{2}(\hat{\Omega})}+\|g\|_{H^{-1}(\hat{\Omega})}+\|h\|_{H^{-1}(\hat{\Omega})}\right)
$$

Finally, returning to the original domain $\Omega$ by the mapping $\Pi^{-1}$, we obtain that $(\rho, \mathbf{u}, \theta)$ is a weak solution to system (2.4)-(2.10), and we can obtain (3.8) from (3.37).

\subsection{Regularity of the weak solutions}

The main result of this section reads as follows.

Theorem 3.4 Assume that all the conditions in Theorem 3.3 are satisfied and $(\mathbf{u}, \rho, \theta) \in$ $H^{1}(\Omega) \times L_{2}(\Omega) \times H^{1}(\Omega)$ is a weak solution to system (2.4)-(2.10) established in Theorem 3.3, then $(\mathbf{u}, \rho, \theta) \in W^{2, p}(\Omega) \times W^{1, p}(\Omega) \times W^{2, p}(\Omega)$, and it holds that

$$
\|\rho\|_{1, p ; \Omega}+\|\mathbf{u}\|_{2, p ; \Omega}+\|\theta\|_{2, p ; \Omega} \leq C\left(\|f\|_{1, p ; \Omega}+\|g\|_{p ; \Omega}+\|h\|_{p ; \Omega}+\left|\rho_{0}\right|_{1, p ;(0,1)}\right)
$$

where $C=C\left(\mu, \lambda, \kappa, p,\|\hat{\mathbf{u}}\|_{2, p ; \Omega}\right)$.

Proof Let $(\mathbf{u}, \rho, \theta)$ be a weak solution established in Theorem 3.3, then $\theta$ satisfies the following boundary problem:

$$
\begin{cases}\triangle \theta=h-\partial_{1} \theta-\operatorname{div} \mathbf{u} \triangleq \bar{h} & \text { in } \Omega, \\ \theta=0 & \text { on } \partial \Omega,\end{cases}
$$

in the weak sense. Thanks to the homogeneous boundary condition $\theta=0$ on $\partial \Omega$, we can make the odd extension of $\theta$ and $\bar{h}$ with respect to $x_{2}=-1,1$. Then, by a similar argument as in the proof of Lemma 3.2, we can improve the regularity of $\theta$ in $\Omega$ including the corners, i.e., we have the following estimate:

$$
\|\theta\|_{2,2 ; \Omega} \leq C\left(\|\bar{h}\|_{2 ; \Omega} \leq\|\mathbf{u}\|_{1,2 ; \Omega}+\|\theta\|_{1,2 ; \Omega}+\|h\|_{L^{2}(\Omega)}\right) .
$$


Then, by (3.8) and the Sobolev imbedding inequality, one has

$$
\|\nabla \theta\|_{p ; \Omega} \leq C\left(\|f\|_{1, p ; \Omega}+\|g\|_{p ; \Omega}+\|h\|_{p ; \Omega}+\left|\rho_{0}\right|_{1, p ;(0,1)}\right)
$$

which implies, by Theorem 2.5 in [28] as well as the structure of linearized momentum equation (2.5), that

$$
\begin{aligned}
\|\rho\|_{1, p ; \Omega}+\|\mathbf{u}\|_{2, p ; \Omega} & \leq C\left(\|f\|_{1, p ; \Omega}+\|g\|_{p ; \Omega}+\|\nabla \theta\|_{p ; \Omega}+\|h\|_{p ; \Omega}+\left|\rho_{0}\right|_{1, p ;(0,1)}\right) \\
& \leq C\left(\|f\|_{1, p ; \Omega}+\|g\|_{p ; \Omega}+\|h\|_{p ; \Omega}+\left|\rho_{0}\right|_{1, p ;(0,1)}\right) .
\end{aligned}
$$

Then, by the same extension above and the $L^{p}$ interior estimate of elliptic equations, one has

$$
\begin{aligned}
\|\theta\|_{2, p ; \Omega} & \leq C\left(\|\bar{h}\|_{p ; \Omega}+\|\mathbf{u}\|_{1, p ; \Omega}+\|\theta\|_{1, p ; \Omega}+\|h\|_{L^{p}(\Omega)}\right) \\
& \leq C\left(\|f\|_{1, p ; \Omega}+\|g\|_{p ; \Omega}+\|h\|_{p ; \Omega}+\left|\rho_{0}\right|_{1, p ;(0,1)}\right)
\end{aligned}
$$

and (3.38) follows from (3.42) and (3.43) immediately.

\section{Proof of Theorem 1.1}

In this section we will prove the main result Theorem 1.1. To this end, we first consider the following solution sequence:

$$
\begin{aligned}
& \partial_{1} \rho^{n+1}+\left(\mathbf{u}^{n}+\tilde{\mathbf{u}}\right) \cdot \nabla \rho^{n+1}+\operatorname{div} \mathbf{u}^{n+1}=F\left(\mathbf{u}^{n}, \rho^{n}\right) \quad \text { in } \Omega, \\
& \partial_{1} \mathbf{u}^{n+1}+\nabla \rho^{n+1}-\nabla \theta^{n+1}-\operatorname{div} \mathbb{S}\left(\nabla \mathbf{u}^{n+1}\right)=G\left(\mathbf{u}^{n}, \rho^{n}, \theta^{n}\right) \quad \text { in } \Omega, \\
& \partial_{1} \theta^{n+1}+\operatorname{div} \mathbf{u}^{n+1}-\kappa \Delta \theta^{n+1}=H\left(\mathbf{u}^{n}, \rho^{n}, \theta^{n}\right) \quad \text { in } \Omega, \\
& \rho^{n+1}=\rho_{\text {in }}-1 \quad \text { on } \Gamma_{\text {in }}, \\
& \mathbf{u}^{n+1}=0 \quad \text { on } \Gamma_{\text {in }} \cup \Gamma_{\text {out }}, \\
& \mathbf{u}^{n+1} \cdot \mathbf{n}=\operatorname{curl} \mathbf{u}^{n+1}=0 \quad \text { on } \Gamma_{0}, \\
& \theta^{n+1}=0 \quad \text { on } \partial \Omega,
\end{aligned}
$$

where $F, G$ and $H$ are defined in $(2.3)$ and $\left(\mathbf{u}^{0}, \rho^{0}, \theta^{0}\right)=(0,0,0)$. By Theorems 3.3 and 3.4, we can obtain a solution sequence $\left\{\left(\mathbf{u}^{n}, \rho^{n}, \theta^{n}\right)\right\}$ in $W^{2, p}(\Omega) \times W^{1, p}(\Omega) \times W^{2, p}(\Omega)$ to system (4.1) with the following estimate:

$$
\begin{gathered}
\left\|\mathbf{u}^{n+1}\right\|_{2, p ; \Omega}+\left\|\rho^{n+1}\right\|_{1, p ; \Omega}+\left\|\theta^{n+1}\right\|_{2, p ; \Omega} \\
\leq C_{1}\left(\left\|F\left(\mathbf{u}^{n}, \rho^{n}\right)\right\|_{1, p ; \Omega}+\left\|G\left(\mathbf{u}^{n}, \rho^{n}, \theta^{n}\right)\right\|_{p ; \Omega}\right. \\
\left.\quad+\left\|H\left(\mathbf{u}^{n}, \rho^{n}, \theta^{n}\right)\right\|_{p ; \Omega}+\left|\rho_{i n}-1\right|_{1, p ; \Gamma_{\text {in }}}\right),
\end{gathered}
$$

where $C_{1}=C_{1}(\mu, \lambda, p, \kappa)$.

Because of the term $\left(\mathbf{u}^{n}+\tilde{\mathbf{u}}\right) \cdot \nabla \rho^{n+1}$ in $(4.1)_{1}$, we are not able to show directly the convergence of the sequence $\left\{\left(\mathbf{u}^{n}, \rho^{n}, \theta^{n}\right)\right\}$ in $W^{2, p}(\Omega) \times W^{1, p}(\Omega) \times W^{2, p}(\Omega)$ to the strong solution of system (2.2) by the fixed point theory. To overcome this, we first show that the 
sequence $\left\{\left(\mathbf{u}^{n}, \rho^{n}, \theta^{n}\right)\right\}$ is bounded uniformly in $W^{2, p}(\Omega) \times W^{1, p}(\Omega) \times W^{2, p}(\Omega)$. Besides, the sequence is also a Cauchy sequence in $H^{1}(\Omega) \times L^{2}(\Omega) \times H^{1}(\Omega)$ and thus converges to some $(\overline{\mathbf{u}}, \bar{\rho}, \bar{\theta})$ in $H^{1}(\Omega) \times L^{2}(\Omega) \times H^{1}(\Omega)$. On the other hand, the uniform bound implies that $(\overline{\mathbf{u}}, \bar{\rho}, \bar{\theta}) \in W^{2, p}(\Omega) \times W^{1, p}(\Omega) \times W^{2, p}(\Omega)$, such that the solution is strong.

Lemma 4.1 Let $\left\{\left(\mathbf{u}^{n}, \rho^{n}, \theta^{n}\right)\right\}$ be the solutions to system (4.1), then it holds that

$$
\left\|\mathbf{u}^{n}\right\|_{2, p ; \Omega}+\left\|\rho^{n}\right\|_{1, p ; \Omega}+\left\|\theta^{n}\right\|_{2, p ; \Omega} \leq M\left(\|\tilde{\mathbf{u}}\|_{2, p ; \Omega}+\left|\rho_{i n}-1\right|_{1, p ; \Gamma_{\text {in }}}+\|\tilde{\theta}\|_{2, p ; \Omega}\right),
$$

where $M>0$ is a constant depending only on $p$.

Proof We will prove (4.3) by an induction argument.

When $n=1$, since $\left(\mathbf{u}^{0}, \rho^{0}, \theta^{0}\right)=(0,0,0)$, we have from (4.2) that

$$
\left\|\mathbf{u}^{1}\right\|_{2, p ; \Omega}+\left\|\rho^{1}\right\|_{1, p ; \Omega}+\left\|\theta^{1}\right\|_{2, p ; \Omega} \leq C_{1}\left(\|\tilde{\mathbf{u}}\|_{2, p ; \Omega}+\|\tilde{\theta}\|_{2, p ; \Omega}+\left|\rho_{\text {in }}-1\right|_{1, p ; \Gamma_{\text {in }}}\right)
$$

and we can obtain (4.3) by choosing $M \geq 4 C_{1}$.

Now, if for $\forall 1 \leq k \leq n$,

$$
\left\|\mathbf{u}^{k}\right\|_{2, p ; \Omega}+\left\|\rho^{k}\right\|_{1, p ; \Omega}+\left\|\theta^{k}\right\|_{2, p ; \Omega} \leq M\left(\|\tilde{\mathbf{u}}\|_{2, p ; \Omega}+\|\tilde{\theta}\|_{2, p ; \Omega}+\left|\rho_{0}-1\right|_{1, p ; \Gamma_{\text {in }}}\right)
$$

and denoting $A_{k}=\left\|\mathbf{u}^{k}\right\|_{2, p ; \Omega}+\left\|\rho^{k}\right\|_{1, p ; \Omega}+\left\|\theta^{k}\right\|_{2, p ; \Omega}$ and $B=\|\tilde{\mathbf{u}}\|_{2, p ; \Omega}+\|\tilde{\theta}\|_{2, p ; \Omega}+\left|\rho_{0}-1\right|_{1, p ; \Gamma_{\text {in }}}$, we obtain by (4.2) and the definition of $F\left(\mathbf{u}^{n}, \rho^{n}\right), G\left(\mathbf{u}^{n}, \rho^{n}, \theta^{n}\right)$ and $H\left(\mathbf{u}^{n}, \rho^{n}, \theta^{n}\right)$ that

$$
A_{n+1} \leq C_{1}\left(A_{n}^{2}+A_{n} B+B\right)
$$

Since $B$ is small enough, we obtain (4.3) immediately.

Lemma 4.2 Let $\left\{\left(\mathbf{u}^{n}, \rho^{n}, \theta^{n}\right)\right\}$ be the solutions to system (4.1), then

$$
\begin{aligned}
& \left\|\mathbf{u}^{n+1}-\mathbf{u}^{n}\right\|_{1,2 ; \Omega}+\left\|\rho^{n+1}-\rho^{n}\right\|_{2 ; \Omega}+\left\|\theta^{n+1}-\theta^{n}\right\|_{1,2 ; \Omega} \\
& \quad \leq \frac{1}{2}\left(\left\|\mathbf{u}^{n}-\mathbf{u}^{n-1}\right\|_{1,2 ; \Omega}+\left\|\rho^{n}-\rho^{n-1}\right\|_{2 ; \Omega}+\left\|\theta^{n}-\theta^{n-1}\right\|_{1,2 ; \Omega}\right) .
\end{aligned}
$$


Proof A straightforward calculation gives

$$
\begin{aligned}
& \partial_{1}\left(\rho^{n+1}-\rho^{n}\right)+\left(\mathbf{u}^{n}+\tilde{\mathbf{u}}\right) \cdot \nabla\left(\rho^{n+1}-\rho^{n}\right)+\operatorname{div}\left(\mathbf{u}^{n+1}-\mathbf{u}^{n}\right) \\
& \quad=F\left(\mathbf{u}^{n}, \rho^{n}\right)-F\left(\mathbf{u}^{n-1}, \rho^{n-1}\right)-\left(\mathbf{u}^{n}-\mathbf{u}^{n-1}\right) \cdot \nabla \rho^{n} \quad \text { in } \Omega, \\
& \partial_{1}\left(\mathbf{u}^{n+1}-\mathbf{u}^{n}\right)+\nabla\left(\rho^{n+1}-\rho^{n}\right)+\nabla\left(\theta^{n+1}-\theta^{n}\right)-\mu \Delta\left(\mathbf{u}^{n+1}-\mathbf{u}^{n}\right) \\
& \quad-\lambda \nabla \operatorname{div}\left(\mathbf{u}^{n+1}-\mathbf{u}^{n}\right) \\
& \quad=G\left(\mathbf{u}^{n}, \rho^{n}, \theta^{n}\right)-G\left(\mathbf{u}^{n-1}, \rho^{n-1}, \theta^{n-1}\right) \quad \text { in } \Omega, \\
& \partial_{1}\left(\theta^{n+1}-\theta^{n}\right)+\operatorname{div}\left(\mathbf{u}^{n+1}-\mathbf{u}^{n}\right)-\kappa \Delta\left(\theta^{n+1}-\theta^{n}\right) \\
& \quad=H\left(\mathbf{u}^{n}, \rho^{n}, \theta^{n}\right)-H\left(\mathbf{u}^{n-1}, \rho^{n-1}, \theta^{n-1}\right) \quad \text { in } \Omega, \\
& \left.\left(\rho^{n+1}-\rho^{n}\right)\right|_{\Gamma_{\text {in }}}=0, \\
& \left.\left(\mathbf{u}^{n+1}-\mathbf{u}^{n}\right)\right|_{\Gamma_{\text {in }} \cup \Gamma_{\text {out }}}=0, \\
& \left.\left(\mathbf{u}^{n+1}-\mathbf{u}^{n}\right) \cdot \mathbf{n}\right|_{\Gamma_{0}}=\left.\operatorname{curl}\left(\mathbf{u}^{n+1}-\mathbf{u}^{n}\right)\right|_{\Gamma_{0}}=0, \\
& \left.\left(\theta^{n+1}-\theta^{n}\right)\right|_{\partial \Omega}=0 .
\end{aligned}
$$

Then, by (4.3) and Lemma 3.3,

$$
\begin{aligned}
&\left\|\mathbf{u}^{n+1}-\mathbf{u}^{n}\right\|_{1,2 ; \Omega}+\left\|\rho^{n+1}-\rho^{n}\right\|_{2 ; \Omega}+\left\|\theta^{n+1}-\theta^{n}\right\|_{1,2 ; \Omega} \\
& \leq C\left[\left\|F\left(\mathbf{u}^{n}, \rho^{n}\right)-F\left(\mathbf{u}^{n-1}, \rho^{n-1}\right)\right\|_{L^{2}}+\left\|G\left(\mathbf{u}^{n}, \rho^{n}, \theta^{n}\right)-G\left(\mathbf{u}^{n-1}, \rho^{n-1}, \theta^{n-1}\right)\right\|_{H^{-1}}\right. \\
&\left.+\left\|H\left(\mathbf{u}^{n}, \rho^{n}, \theta^{n}\right)-H\left(\mathbf{u}^{n-1}, \rho^{n-1}, \theta^{n-1}\right)\right\|_{H^{-1}}+\left\|\left(\mathbf{u}^{n}-\mathbf{u}^{n-1}\right) \cdot \nabla \rho^{n}\right\|_{L^{2}}\right] \\
& \leq C\left(A_{n}+A_{n-1}+B\right)\left(\left\|\mathbf{u}^{n}-\mathbf{u}^{n-1}\right\|_{1,2 ; \Omega}+\left\|\rho^{n}-\rho^{n-1}\right\|_{2 ; \Omega}+\left\|\theta^{n}-\theta^{n-1}\right\|_{1,2 ; \Omega}\right) \\
& \leq \frac{1}{2}\left(\left\|\mathbf{u}^{n}-\mathbf{u}^{n-1}\right\|_{1,2 ; \Omega}+\left\|\rho^{n}-\rho^{n-1}\right\|_{2 ; \Omega}+\left\|\theta^{n}-\theta^{n-1}\right\|_{1,2 ; \Omega}\right),
\end{aligned}
$$

thus the lemma follows consequently.

Proof of Theorem 1.1 Lemma 4.2 implies that $\left\{\left(\mathbf{u}^{n}, \rho^{n}, \theta^{n}\right)\right\}$ is a Cauchy sequence in $H^{1}(\Omega) \times L^{2}(\Omega) \times H^{1}(\Omega)$. Thus there is unique $(\overline{\mathbf{u}}, \bar{\rho}, \bar{\theta}) \in H^{1}(\Omega) \times L^{2}(\Omega) \times H^{1}(\Omega)$ such that

$$
\lim _{n \rightarrow \infty}\left[\left\|\mathbf{u}^{n}-\overline{\mathbf{u}}\right\|_{1,2 ; \Omega}+\left\|\rho^{n}-\bar{\rho}\right\|_{2 ; \Omega}+\left\|\theta^{n}-\bar{\theta}\right\|_{1,2 ; \Omega}\right]=0 .
$$

On the other hand, in Lemma 4.1, we have proved that $\left\{\left(\mathbf{u}^{n}, \rho^{n}, \theta^{n}\right)\right\}$ have a uniform bound in $W^{2, p}(\Omega) \times W^{1, p}(\Omega) \times W^{2, p}(\Omega)$, which implies combined with (4.6) that $(\overline{\mathbf{u}}, \bar{\rho}, \bar{\theta})$ is also in $W^{2, p}(\Omega) \times W^{1, p}(\Omega) \times W^{2, p}(\Omega)$, and the sequence $\left\{\left(\mathbf{u}^{n}, \rho^{n}, \theta^{n}\right)\right\}$ converges strongly to $(\overline{\mathbf{u}}, \bar{\rho}, \bar{\theta})$ in $W^{2, p^{\prime}}(\Omega)$ for $\forall 1<p^{\prime}<p$.

Now we can take limit on both sides of (4.1) and conclude that $(\overline{\mathbf{u}}, \bar{\rho}, \bar{\theta})$ satisfies system (2.2), i.e., $\mathbf{u}=\overline{\mathbf{u}}+\tilde{\mathbf{u}}+U_{0}, \rho=\bar{\rho}+1, \theta=\bar{\theta}+\tilde{\theta}+1$ satisfy system (1.5). Moreover, estimate (1.7) follows from (2.1) and (4.3) immediately.

Finally, if $(\mathbf{u}, \rho, \theta)$ and $(\hat{\mathbf{u}}, \hat{\rho}, \hat{\theta})$ are two solutions of system (1.5), then by a process similar to that used in Lemma 4.2, we have

$$
\|\mathbf{u}-\hat{\mathbf{u}}\|_{1,2 ; \Omega}+\|\rho-\hat{\rho}\|_{2 ; \Omega}+\|\theta-\hat{\theta}\|_{1,2 ; \Omega} \leq \frac{1}{2}\left(\|\mathbf{u}-\hat{\mathbf{u}}\|_{1,2 ; \Omega}+\|\rho-\hat{\rho}\|_{2 ; \Omega}+\|\theta-\hat{\theta}\|_{1,2 ; \Omega}\right) .
$$


Consequently, we obtain the uniqueness of the solutions. This completes the proof of Theorem 1.1.

\section{Conclusions}

In this paper, we have established strong solutions to the steady compressible heatconductive fluid near a non-zero constant flow with the Dirichlet boundary condition for the velocity on the inflow and outflow part of the boundary, and the Dirichlet boundary condition for temperature on $\partial \Omega$. For our result, we do not need the Reynolds number and the Mach number to be small and do not need the thermal conductivity coefficient $\kappa$ to be large. To the best of our knowledge, it is first result to establish the strong solutions to the steady compressible heat-conductive fluid near a non-zero constant flow considering the Dirichlet boundary condition for the velocity on the inflow part of the boundary without the smallness assumption of the Reynolds number and the Mach number.

\section{Funding}

This work is supported by NSFC 11101043.

\section{List of abbreviations}

Not applicable

Availability of data and materials

Not applicable

Ethics approval and consent to participate

Not applicable

Competing interests

The author declares that they have no competing interests.

Consent for publication

Not applicable

Author's contributions

All authors read and approved the final manuscript.

\section{Publisher's Note}

Springer Nature remains neutral with regard to jurisdictional claims in published maps and institutional affiliations.

Received: 13 September 2017 Accepted: 9 November 2017 Published online: 22 November 2017

\section{References}

1. Feireisl, E: Dynamics of Viscous Compressible Fluids. Oxford University Press, Oxford (2004)

2. Jesslé, D, Novotný, A: Existence of renormalized weak solutions to the steady equations describing compressible fluids in barotropic regime. J. Math. Pures Appl. 99, 280-296 (2013)

3. Jiang, S, Zhou, C: Existence of weak solutions to the three-dimensional steady compressible Navier-Stokes equations. Ann. Inst. Henri Poincaré, Anal. Non Linéaire 28, 485-498 (2011)

4. Lions, PL: Mathematical Topics in Fluid Mechanics. Compressible Models, vol. Il. Clarendon Press, Oxford (1998)

5. Jesslé, D, Novotný, A, Pokorny, M: Steady Navier-Stokes-Fourier system with slip boundary conditions. Math. Models Methods Appl. Sci. 24(4), 751-781 (2014)

6. Mucha, PB, Pokorný, M: On the steady compressible Navier-Stokes-Fourier system. Commun. Math. Phys. 288, 349-377 (2009)

7. Mucha, PB, Pokorny, M: Weak solutions to equations of steady compressible heat conducting fluids. Math. Models Methods Appl. Sci. 20(5), 785-813 (2010)

8. Novotný, A, Pokorný, M: Steady compressible Navier-Stokes-Fourier system for monoatomic gas and its generalizations. J. Differ. Equ. 251, 270-315 (2011)

9. Novotný, A, Pokorný, M: Weak and variational solutions to steady equations for compressible heat conducting fluids. SIAM J. Math. Anal. 43, 270-315 (2011)

10. Plotnikov, PI, Weigant, W: Steady $3 \mathrm{D}$ viscous compressible flows with adiabatic exponent $\gamma \in(1, \infty)$. J. Math. Pures Appl. 104, 58-82 (2015)

11. Ait Mahiout, L, Amaziane, B, Mokrane, A, Pankratov, L: Homogenization of immiscible compressible two-phase flow in double porosity media. Electron. J. Differ. Equ. 2016, 52 (2016)

12. Choe, $H$, Jin, B: Existence of solutions of stationary compressible Navier-Stokes equations with large force. J. Funct. Anal. 177, 54-88 (2000) 
13. Denny, D: Existence of solutions to systems of equations modelling compressible fluid flow. Electron. J. Differ. Equ. 2015, $216(2015)$

14. Dou, C, Jiang, F, Jiang, S, Yang, Y: Existence of strong solutions to the steady Navier-Stokes equations for a compressible heat-conductive fluid with large forces. J. Math. Pures Appl. 103, 1163-1197 (2015)

15. Nazarov, SA, Novotný, A, Pileckas, K: On steady compressible Navier-Stokes equations in plane domains with corners. Math. Ann. 304, 121-150 (1996)

16. Padula, M: Existence and uniqueness for viscous steady compressible motions. Arch. Ration. Mech. Anal. 77, 89-102 (1987)

17. Valli, A: On the existence of stationary solutions to compressible Navier-Stokes equations. Ann. Inst. Henri Poincaré 4 , 99-113 (1987)

18. Valli, A, Zajaczkowski, WM: Navier-Stokes equations for compressible fluids: global existence and qualitative properties of the solutions in the general case. Commun. Math. Phys. 103, 259-296 (1986)

19. Padula, M: Steady flows of barotropic viscous fluids. In: Classical Problems in Mechanics, vol. 1, pp. 253-345 (1997)

20. Kellogg, RB, Kweon, JR: Compressible Navier-Stokes equations in a bounded domain with inflow boundary condition. SIAM J. Math. Anal. 28, 94-108 (1997)

21. Kweon, JR, Kellogg, RB: Regularity of solutions to the Navier-Stokes equations for compressible barotropic flows on a polygon. Arch. Ration. Mech. Anal. 163, 35-64 (2002)

22. Kweon, JR: A jump discontinuity of compressible viscous flows grazing a non-convex corner. J. Math. Pures Appl. 100 410-432 (2013)

23. Kweon, JR, Kellogg, RB: Regularity of solutions to the Navier-Stokes system for compressible flows on a polygon. SIAM J. Math. Anal. 35, 1451-1485 (2004)

24. Plotnikov, PI, Ruban, EV, Sokolowski, J: Inhomogeneous boundary value problems for compressible Navier-Stokes equations: well-posedness and sensitivity analysis. SIAM J. Math. Anal. 40, 1152-1200 (2008)

25. Plotnikov, Pl, Ruban, EV, Sokolowski, J: Inhomogeneous boundary value problems for compressible Navier-Stokes and transport equations. J. Math. Pures Appl. 92, 113-162 (2009)

26. Piasecki, T: On an inhomogeneous slip-inflow boundary value problem for a steady flow of a viscous compressible fluid in a cylindrical domain. J. Differ. Equ. 248, 2171-2198 (2010)

27. Piasecki, T, Pokorný, M: Strong solutions to the Navier-Stokes-Fourier system with slip-inflow boundary conditions. Z. Angew. Math. Mech. 94, 1035-1057 (2014)

28. Guo, Y, Jiang, S, Zhou, C: Steady viscous compressible channel flows. SIAM J. Math. Anal. 47(5), 3648-3670 (2015)

29. Gilbarg, D, Trudinger, NS: Elliptic Partial Differential Equations of Second Order, 2nd edn. Springer, Berlin (1983)

\section{Submit your manuscript to a SpringerOpen ${ }^{\circ}$ journal and benefit from:}

- Convenient online submission

- Rigorous peer review

- Open access: articles freely available online

- High visibility within the field

- Retaining the copyright to your article

Submit your next manuscript at $\gg$ springeropen.com 